\title{
Evaluación de carotenoides y lípidos en la microalga Scenedesmus dimorphus a escala laboratorio
}

\section{Evaluation of Carotenoids and lipids in the Microalga Scenedesmus dimorphus at Laboratory Scale}

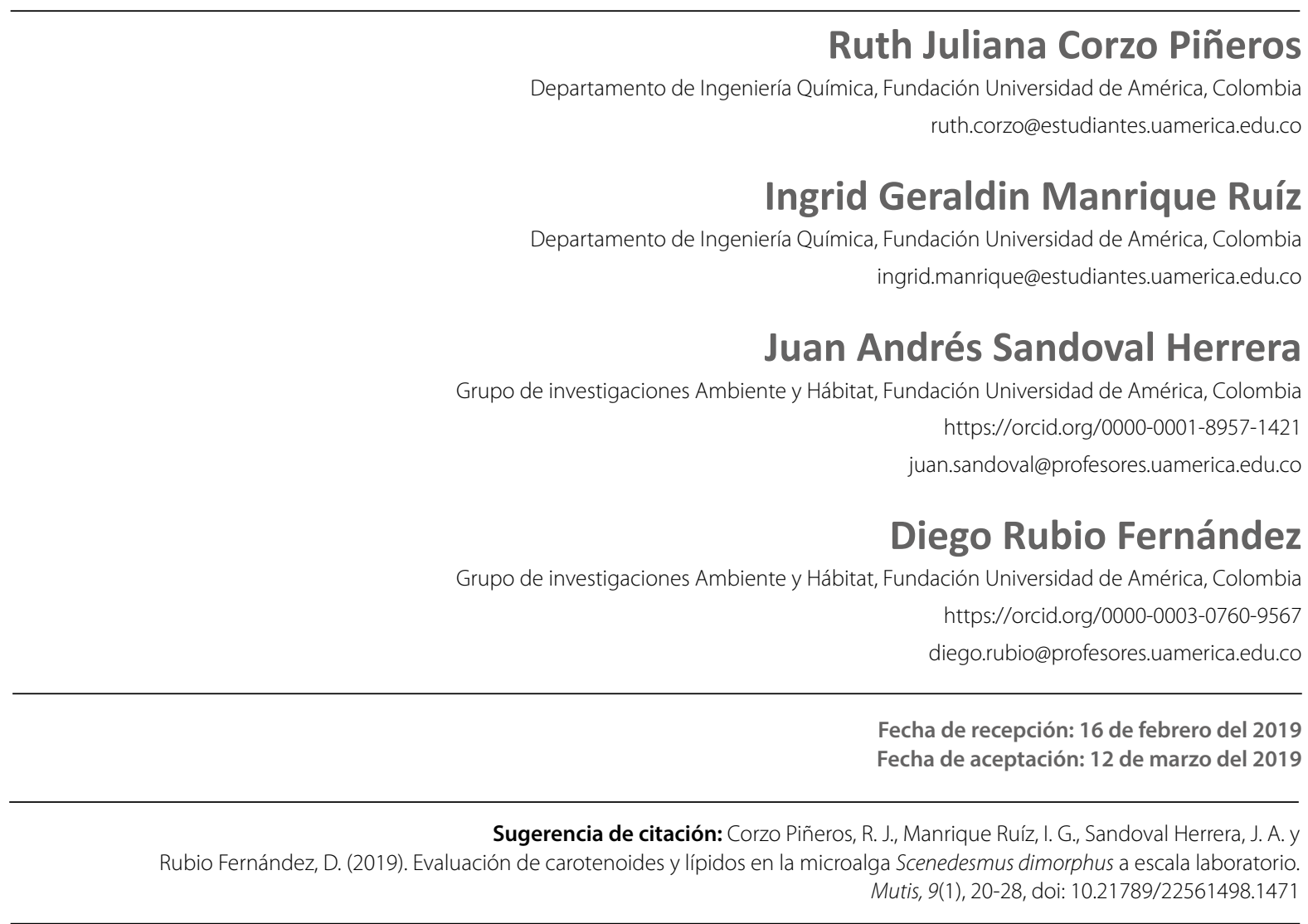

\section{RESUMEN}

Los carotenoides son metabolitos producidos por microalgas que cumplen la función de pigmentos accesorios en la fotosíntesis y de moléculas fotoprotectoras. Con el fin de incrementar la acumulación de los carotenoides en la microalga Scenedesmus dimorphus, se evaluó la salinidad $\left(\mathrm{NaCl}\right.$ y $\mathrm{KNO}_{3}$ ) y la luz LED azul como factores de estrés en la fase estacionaria del crecimiento del cultivo de esta alga. De las dos sales estudiadas la mejor fue $\mathrm{KNO}_{3}$ $0,6 \mathrm{M}$, puesto que el cultivo alcanzó la mayor concentración de carotenoides totales $(0,3357 \pm 0,02 \mu \mathrm{g}$ de carotenoides $\mathrm{mL}^{-1}$ ), en contraste con 0,1791 $\pm 0,01 \mu \mathrm{g} \mathrm{mL}{ }^{-1}$ del cultivo $\mathrm{NaCl} 0,6 \mathrm{M}$. Posteriormente, se escaló el proceso en un recipiente adaptado de $4 \mathrm{~L}$, alcanzando fase estacionaria en el día 12 y adicionando el $\mathrm{KNO}_{3} 0,6 \mathrm{M}$ el día 14 ; 
en el día 20 (final del cultivo) se obtuvo mediante espectrofotometría un total de $0,7395 \pm 0,05 \mu \mathrm{g}$ de carotenoides $\mathrm{mL}^{-1}$. Seguido, se realizó el secado del cultivo con salinidad, obteniendo un peso seco de $5,7 \mathrm{~g}$ y una productividad de $0,0927 \mathrm{~g} \mathrm{~L}^{-1} \mathrm{~d}^{-1}$. Finalmente, a través de cromatografía HPLC, se analizó el perfil lipídico del cultivo, obteniendo $21,1 \%$ de MUFAS, $35,9 \%$ de SFAS Y $43 \%$ de PUFAS (omega- 6 de $34 \%$ y omega-3 de $8,9 \%$ ). Con base en estos resultados, se determinó que la salinidad es un posible factor para incrementar en mayor proporción la concentración de lípidos que de carotenoides, específicamente en una proporción de 1:2.500 de carotenoides y lípidos, respectivamente.

Palabras clave: microalga, biomasa, carotenoides, lípidos, salinidad.

\section{ABSTRACT}

Carotenoids are metabolites produced by microalgae that perform as accessory pigments in photosynthesis and as photoprotective molecules. In order to increase the accumulation of carotenoids in the microalga Scenedesmus dimorphus, salinity $(\mathrm{NaCl}$ and $\mathrm{KNO}_{3}$ ) and blue LED light were evaluated as stress factors when reaching the stationary phase of culture growth in such microalga. After evaluation, the best of the two salts studied was $\mathrm{KNO}_{3} 0.6 \mathrm{M}$, since this culture reached the highest concentration of total carotenoids $\left(0.3357 \pm 0.02 \mu \mathrm{g}\right.$ carotenoids $\left.\mathrm{mL}^{-1}\right)$, in contrast to $0.1791 \pm 0.01 \mu \mathrm{g} \mathrm{mL}^{-1}$ with $\mathrm{NaCl} 0.6 \mathrm{M}$. Subsequently, the process was scaled in an adapted container of 4 $\mathrm{L}$, entering the stationary phase on day 12 . On day 14 the $\mathrm{KNO}_{3}$ was added at $0.6 \mathrm{M}$; this experiment lasted 20 days of cell count, obtaining on the last day a total of $0,7395 \pm 0,05 \mu \mathrm{g}$ of carotenoids $\mathrm{mL}^{-1}$ by means of spectrophotometry. Afterwards, drying of the culture with salinity was carried out, obtaining a dry weight of $5.7 \mathrm{~g}$, with a productivity of $0.0927 \mathrm{~g} \mathrm{~L}^{-1} \mathrm{~d}^{-1}$. Finally, through HPLC chromatography, the lipid profile of the culture was analyzed, resulting in $21.1 \%$ MUFAS, $35.9 \%$ SFAS, and $43 \%$ PUFAS (34\% omega- 6 and $8.9 \%$ omega-3). Based on the above, it was determined that salinity is a possible factor to increase in a greater proportion the concentration of lipids than that of carotenoids, specifically in a ratio of 1:2,500 carotenoids and lipids, respectively.

Keywords: Microalga, biomass, carotenoids, lipids, salinity.

\section{INTRODUCCIÓN}

A través del tiempo se han buscado alternativas para sustituir materias primas cuyo uso no se considera adecuado; bien sea por factores como costos, generación de residuos, efectos en la salud, entre otros. Un claro ejemplo es el empleo de pigmentos alimenticios sintéticos que ocasionan problemas de salud tales como alergias, complicaciones digestivas e incluso cáncer (Velázquez, 2012). Por esta razón, reemplazar dichos pigmentos por pigmentos naturales se ha convertido en una necesidad. Una de las posibles alternativas para dicha sustitución son los carotenoides provenientes de microalgas, los cuales corresponden a lípidos simples, específicamente terpenos (Plaza, Herrero, Cifuentes, \& Ibáñez, 2009). Sin embargo, para conseguir una producción viable de estos compuestos es importante seleccionar el factor de estrés con mayores beneficios para el proceso a nivel de productividad y costos, así como realizar una adecuada selección de la cepa.

Por consiguiente, el objetivo de este trabajo fue evaluar el potencial de producción de carotenoides de la microalga Scenedesmus dimorphus en un recipiente adaptado bajo una condición experimental seleccionada, así como establecer la proporción de carotenoides-lípidos producidos bajo un factor de estrés determinado.

\section{MATERIALES Y MÉTODOS}

\section{Selección de factor de estrés}

En primera instancia, se llevó a cabo una revisión bibliográfica de publicaciones de los últimos diez años sobre los factores que tienen mayor efecto en la inducción de carotenoides en la microalga Scenedesmus dimorphus. Para ello, se creó una matriz de selección (tabla 1) que incluía estrategias a nivel nutricional; es decir, aquellas en las que se aplican cambios en la composición del medio de cultivo, como la limitación de nitrógeno, fosfato y azufre, o a nivel físico, por la manipulación de las condiciones operacionales y factores externos como luz, salinidad y $\mathrm{pH}$. En esta matriz de selección se evaluaron parámetros como la viabilidad de aplicación según sistemas de instrumentación y control, tiempo de duración del experimento, costos en la aplicación del factor, especie de microalga, productividad de carotenoides 
y factor escala. Posteriormente, a partir del puntaje obtenido, se seleccionó como factor de estrés la salinidad, teniendo como referente las condiciones pre- sentes en el Laboratorio de la Fundación Universidad de América (Bogotá).

Tabla 1. Matriz de selección del factor de estrés

\begin{tabular}{|c|c|c|c|c|c|c|}
\hline $\begin{array}{l}\text { Factor de } \\
\text { estrés }\end{array}$ & $\begin{array}{l}\text { Viabilidad de } \\
\text { aplicación }\end{array}$ & $\begin{array}{l}\text { Tiempo } \\
\text { (días) }\end{array}$ & Costos & $\begin{array}{l}\text { Especie } \\
\text { microalga }\end{array}$ & $\begin{array}{l}\text { Productividad de } \\
\text { carotenoides }\end{array}$ & Referencias \\
\hline Luz & $\begin{array}{l}\text { Luz LED ( } 75 \% \text { roja } \\
\text { y } 25 \% \text { azul) y } \\
\text { sensor cuántico }\end{array}$ & 5 & $\begin{array}{l}\text { Sensor cuántico } \\
\text { QUANTUM, Modelo LI- } \\
\text { 1400, COP } 360.000\end{array}$ & Dunaliella salina & $\begin{array}{c}\text { Luteína }(7,9 \\
\left.\text { mg g }^{-1} \text { DCW }\right) \text { y } \\
\text { Betacaroteno }\left(9,5 \mathrm{mg} \mathrm{g}^{-1}\right. \\
\text { DCW })\end{array}$ & $\begin{array}{c}\text { Fu et al. (2013) } \\
\text { Wichuk et al. (2014) }\end{array}$ \\
\hline $\begin{array}{l}\text { Limitación de } \\
\text { nitrógeno }\end{array}$ & $\begin{array}{c}\text { Medio } f / 2 \text { de } \\
\text { Guillard y Ryther a } \\
1 \text { L de agua de mar } \\
\text { filtrada }\end{array}$ & $7-10$ & $\begin{array}{c}\text { El medio } \mathrm{f} / 2 \mathrm{de} \\
\text { Guillard y Ryther, cop } \\
190.000\end{array}$ & $\begin{array}{l}\text { Dunaliella } \\
\text { tertiolecta }\end{array}$ & $\begin{array}{c}\text { 0,03 } \mathrm{mg} \mathrm{L}^{-1} \text { de zeaxantina, } \\
9,19 \mathrm{mg} \mathrm{L}^{-1} \text { de luteína y } \\
5,40 \mathrm{mg} \mathrm{L}^{-1} \text { de } \beta \text {-caroteno }\end{array}$ & López et al. (2013) \\
\hline $\begin{array}{l}\text { Limitación } \\
\text { de fosfato y } \\
\text { azufre }\end{array}$ & $\begin{array}{l}\text { Medio } f / 2 \\
\text { modificado }\end{array}$ & 5 & $\begin{array}{c}\text { El medio } f / 2 \text { de } \\
\text { Guillard y Ryther, cop } \\
190.000\end{array}$ & Nannochloropsis & $\begin{array}{l}\text { Carotenoides totales } \\
\text { obtenidos } 20 \mathrm{ng} \times 10^{-6} \\
\text { células }\end{array}$ & $\begin{array}{l}\text { Forján-Lozano et al. } \\
\qquad(2007)\end{array}$ \\
\hline $\mathrm{pH}$ & $\begin{array}{c}\text { Los cultivos se } \\
\text { incubaron a } 25^{\circ} \mathrm{C}\end{array}$ & 35 & $\begin{array}{l}\text { Medio de agua de mar } \\
\text { artificial, COP } 190.000\end{array}$ & Dunaliella salina & $\begin{array}{c}5,21 \mu \mathrm{g} \text { de carotenoides } \\
\mathrm{mL}^{-1}, \text { a pH } 9\end{array}$ & Dhaka y Singh (2018) \\
\hline Salinidad & $\mathrm{NaCl}$ & 23 & $\mathrm{NaCl}, \operatorname{COP} 20.000 / \mathrm{kg}$ & Dunaliella salina & $\begin{array}{c}\text { A concentración salina de } \\
300 \text { uPs, la productividad } \\
\text { de carotenoides fue } 6,1 \\
\mathrm{mg} \mathrm{L}^{-1} \text {. }\end{array}$ & López (2008) \\
\hline
\end{tabular}

\section{Condiciones del pre-experimento}

Se evaluaron dos tipos de sal $\left(\mathrm{NaCl}\right.$ y $\left.\mathrm{KNO}_{3}\right)$ a concentraciones de $0,5 \mathrm{M} ; 0,6 \mathrm{M} ; 0,7 \mathrm{M}$ y $0,8 \mathrm{M}$.

\section{Montaje del pre-experimento}

Para esta fase del experimento, se pusieron en marcha nueve cultivos con volumen de trabajo de $300 \mathrm{~mL}$, de los cuales cuatro correspondían a cultivos para adicionar cloruro de sodio y otros cuatro para adicionar nitrato de potasio. Esta adición se realizó al presenciar el inicio de la fase estacionaria de los cultivos en la curva de crecimiento realizada, a través de los conteos diarios por cámara de Neubauer. Al último cultivo, denominado blanco, no se le indujo ningún factor de estrés. De los $300 \mathrm{~mL}, 250 \mathrm{~mL}$ correspondían a medio de cultivo Foliagro (tabla 2) y 50 a microalga Scenedesmus dimorphus con un inóculo inicial de $4,03 \times 10^{5} \mathrm{cel} \mathrm{mL}^{-1}$. El medio de cultivo se preparó siguiendo la proporción de $2 \mathrm{~mL}$ de Foliagro por litro de agua destilada.
Tabla 2. Composición del medio de cultivo

\begin{tabular}{ll}
\hline Elemento & Composición $\left(\mathrm{g} \mathrm{L}^{-1}\right)$ \\
\hline Nitrógeno total & 100 \\
Fósforo asimilable & 300 \\
Potasio soluble en agua & 100 \\
Calcio & 0,20 \\
Magnesio & 0,20 \\
Azufre total & 0,80 \\
Boro & 0,20 \\
Cobre & 0,20 \\
Hierro & 0,40 \\
Manganeso & 0,40 \\
Molibdeno & 0,03 \\
Zinc & 0,50 \\
\hline
\end{tabular}

Fuente: fabricantes. 
A continuación, los recipientes fueron dispuestos en una incubadora bajo condiciones axénicas para favorecer el crecimiento de los cultivos. La aireación se realizó por medio de bombas de acuario de marca Jeneca de cuatro salidas cada una, manteniendo un caudal de aire de $4 \mathrm{~L} \mathrm{~min}{ }^{-1}$, las cuales se encontraban conectadas a mangueras de 3/16" que llegaban hasta los recipientes adaptados, permitiendo así una agitación continua -complementada, en ocasiones, con agitación manual- para evitar la sedimentación del cultivo. Siguiendo la recomendación de Rubio, Sandoval, Ruíz, \& Tovar (2017), se empleó luz roja a 680 $\mathrm{nm}$ a lo largo de la incubadora por medio de cintas LED, con una intensidad promedio de $12,1 \mathrm{~W} \mathrm{~m}^{-2}$. Sin embargo, al llegar a fase estacionaria se aplicó solo luz azul para la generación de carotenoides. Por otro lado, se fijó un fotoperiodo de 12 horas de luz y 12 de oscuridad, controlado por un temporizador, para que el cultivo no se viera afectado por fotoinhibición. La temperatura se mantuvo en condiciones del ambiente del laboratorio, aproximadamente $20^{\circ} \mathrm{C}$.

\section{Crecimiento celular}

Por medio del conteo por cámara de Neubauer en cinco cuadrantes se calculó la concentración celular aplicando la siguiente ecuación (Murcia \& Parra, 2018):

$$
\begin{gathered}
\text { Concentración }(\text { cel } / \mathrm{mL})=\text { \#células totales *Factor di- } \\
\text { lución*50.000 (Ec. 1) }
\end{gathered}
$$

Por su parte, la velocidad de crecimiento $(\mu)$ y el tiempo de duplicación $\left(\mathrm{t}_{\mathrm{g}}\right)$ fueron calculados utilizando las ecuaciones 2 y 3 , respectivamente, donde $X_{1}$ y $X_{2}$ son las concentraciones a los tiempos 1 y $2\left(t_{1,2}\right)$ (Vega \& Voltolina, 2007)

$$
\begin{array}{r}
\mu_{e}\left(d^{-1}\right)=\frac{\operatorname{Ln}\left(\frac{X_{2}}{X_{1}}\right)}{t_{2}-t_{1}} \\
t_{g}(\text { dia })=\frac{\operatorname{Ln}(2)}{\mu_{e}}
\end{array}
$$

\section{Cultivo con salinidad}

El conteo celular en cámara de Neubauer se llevó a cabo diariamente para evidenciar, a través de la curva de crecimiento, el día en que se llegó a fase estacionaria (día 7). Las sales fueron agregadas a los ocho recipientes adaptados en el día diez, obteniendo concentraciones de 0,$5 ; 0,6 ; 0,7$ y $0,8 \mathrm{~N}$.

\section{Cuantificación de carotenoides y clorofilas por espectrofotometría UV-VIS}

Para la extracción de pigmentos liposolubles se siguió el protocolo de los investigadores Strickland y Parsons (1972) y Jeffrey y Humphrey (1975), el que describe que para la extracción de los pigmentos se debe separar el medio de cultivo de la biomasa, lo cual se realizó mediante centrifugación a 3.200 rpm en centrífuga Baby I 206 por un periodo de 15 minutos. Al concluir la centrifugación se desechó el sobrenadante mediante pipeta Pasteur. La pastilla celular resultante fue dispuesta en un tubo de vidrio limpio con tapón de rosca, el cual fue cubierto para evitar la foto-oxidación. A este se añadieron $3 \mathrm{~mL}$ de acetona al $90 \%$, agitando para lograr la resuspensión de la pastilla celular y la extracción de los pigmentos. Los tubos con el extracto se dejaron reposar durante 24 horas continuas en baño de hielo. Luego, se realizó centrifugación a 3.200 rpm por 15 minutos, seguida de separación del extracto de pigmentos con la debida protección de la luz. Finalmente, se leyó su absorbancia en un espectrofotómetro previamente calibrado a longitud de onda de 480 y $510 \mathrm{~nm}$ para los carotenoides totales y a 664 y $647 \mathrm{~nm}$ para clorofilas. Con el fin de calcular la concentración de las clorofilas y los carotenoides totales, expresada en xpr $^{-1}$ de extracto, se usaron las ecuaciones propuestas en el protocolo descrito por Vega y Voltolina (2007), donde $A$ es la absorbancia de la muestra:

$$
\begin{aligned}
& \begin{array}{l}
\text { Concentración Clorofila } a=11,93 A_{664}-1,93 A_{647} \\
\text { Conc.6) }
\end{array} \\
& \begin{array}{l}
\text { Concentración Clorofila } b=20,36 A_{647}-5,50 A_{664} \quad \text { (Ec.7) } \\
\text { Cación Carotenos }=7,6\left(A_{480}-1,49 A_{510} \quad(\text { Ec.8) }\right.
\end{array}
\end{aligned}
$$

Por último, con el objetivo de obtener la concentración en $\mu \mathrm{g}$ de carotenoides $\mathrm{mL}^{-1}$ de cultivo, los resultados obtenidos en las ecuaciones fueron multiplicados por el volumen del extracto y divididos por el volumen de muestra.

\section{Diseño factorial}

Mediante el software Excel, se planteó un análisis por diseño factorial $2^{4}$, siendo los factores los dos tipos de sal ( $\mathrm{NaCl}$ y $\mathrm{KNO}_{3}$ ) y los niveles las concentraciones de $0,5 \mathrm{M} ; 0,6 \mathrm{M} ; 0,7 \mathrm{M}$ y $0,8 \mathrm{M}$. Además, se formuló la hipótesis nula, HO: la concentración de sal no genera 
un cambio significativo en la concentración de carotenoides totales, y la hipótesis alternativa, $\mathrm{H} 1$ : la concentración de sal genera un cambio significativo en la concentración de carotenoides totales.

\section{Condiciones del experimento}

El experimento se llevó a cabo en dos recipientes adaptados de $4 \mathrm{~L}$, con un volumen de trabajo total de $3.535 \mathrm{~mL}$, de los cuales $3 \mathrm{~L}$ eran del medio de cultivo y $535 \mathrm{~mL}$ de microalga, con un inoculo inicial de $2 \times 106$ cel $\mathrm{mL}^{-1}$. Un recipiente correspondía al blanco y el otro al cultivo con el factor de estrés. Por medio de los conteos por cámara de Neubauer se determinó el día de inicio de la fase estacionaria (día 12). En el día 14 se agregaron 138,92 gramos de $\mathrm{KNO}_{3}$ a un volumen de $2.290 \mathrm{~mL}$ para obtener la concentración de 0,6 N. Se esperaron 6 días de conteo después de aplicada la sal para observar si se presentaba o no viraje de color (siendo indicativo de presencia de carotenoides), lo cual fue ratificado mediante espectrofotometría. Además, se aplicaron las mismas condiciones operacionales del pre-experimento (longitud de onda, temperatura, velocidad de agitación).

\section{Secado de la biomasa y productividad}

La biomasa fue concentrada en 12 tubos de $14 \mathrm{~mL}$ cada uno, retirando la mayor cantidad de medio de cultivo posible a través del uso de una centrifuga Baby I 206 a 3.200 rpm por 5 minutos. El sobrenadante fue separado, recolectando $648 \mathrm{~mL}$ de pastilla microalgal en 4 moldes desechables de aluminio. Los moldes con $162 \mathrm{~mL}$ de biomasa cada uno fueron dispuestos en una estufa Nabertherm (TR 120) por 18 horas a 60 ${ }^{\circ} \mathrm{C}$, con el fin de retirar la mayor cantidad de humedad posible y conservar la calidad de la muestra. Finalmente, se calculó el peso seco y la productividad con base en las ecuaciones 9 y 10 (Vega \&Voltolina, 2007):$$
\begin{aligned}
& \text { Peso seco }(g)=\frac{\text { Peso seeo fatro (con muestra-sin muestra) }}{\text { Voimmen de muestra fittrado }} \quad \text { (Ec. 9) } \\
& \text { Productividad }\left[g L^{-1} d^{-1}\right]=\frac{\text { Peso seco de Biomasa }}{\text { Voliumen de cultivo-I de Dies }} \quad \text { (Ec. 10) }
\end{aligned}
$$

\section{Cromatografía HPLC}

La muestra seca fue entregada al Laboratorio de Toxicología de la Universidad Nacional de Colombia, donde se realizó la extracción de lípidos y la determina- ción del perfil de ácidos grasos mediante el Método Folch, con el objetivo de plantear la correspondiente proporción carotenoides-lípidos (Restrepo, Díaz, \& Pardo, 2012).

\section{RESULTADOS Y DISCUSIÓN}

\section{Resultados del pre-experimento}

En la figura 1 se representan las fases de crecimiento celular observadas gracias al conteo diario. En los primeros tres días se dio la fase de adaptación; del día 3 al 7 el crecimiento exponencial; y del día 7 al 10 se alcanzó la fase estacionaria. Debido a que se buscaba favorecer una mayor acumulación de carotenoides, la aplicación del estrés salino se realizó en el día 10, mientras el cultivo estaba aún en fase estacionaria (Fernández-Cordero, 2013), observando luego una variación en la concentración celular de los cultivos dependiente de la concentración salina y la sal aplicada en cada recipiente adaptado.

En cuanto a los cultivos adicionados con $\mathrm{NaCl} 0,5 \mathrm{M}$, $0,6 \mathrm{M}$ y $0,7 \mathrm{M}$, se observó un aumento significativo de la concentración celular, alcanzando un máximo de $1,8 \times 10^{7} \pm 25,46 \mathrm{cel} \mathrm{mL}^{-1}$. No se presentó muerte celular. Se observó, además, un cambio de morfología (figura 2), siendo inicialmente delgada y alargada, pero circular después de la aplicación de la sal. Esto se debe a que las altas concentraciones de nutrientes, producen un aumento en la relación superficie/volumen (Acevedo \& Ramírez, 2003). Sin embargo, en el cultivo con $\mathrm{NaCl} 0,8 \mathrm{M}$ no aumentó la concentración celular del cultivo; en cambio, ocurrió fase de muerte del día 13 al 14, lo que indicaría, como señalan Moronta, Mora y Morales (2006), que esta concentración es inhibitoria del crecimiento celular.

Por otro lado, al adicionar $\mathrm{KNO}_{3}$ a las mismas concentraciones de $0,5 \mathrm{M}, 0,6 \mathrm{M}$ y 0,7 M, la concentración celular no presentó crecimiento significativo, alcanzando un máximo de $8,15 \times 10^{6} \pm 8,49 \mathrm{cel} \mathrm{mL}^{-1}$. Los cultivos siguieron en fase estacionaria durante los días posteriores a la adición de la sal, lo que podría deberse a que la microalga estaba sometida a un estrés salino mayor que con el $\mathrm{NaCl}$. Finalmente, la concentración $0,8 \mathrm{M}$ de $\mathrm{KNO}_{3}$ también indujo fase de muerte del día 12 al 14. Con base en estos resultados, se estableció un periodo de dos días después de alcanzar la fase estacionaria para agregar la salinidad; lo ante- 
rior con el fin de prevenir la muerte celular, dado que se empleó una concentración alta, como se verá más adelante.

Figura 1. Curva de crecimiento celular pre-experimento

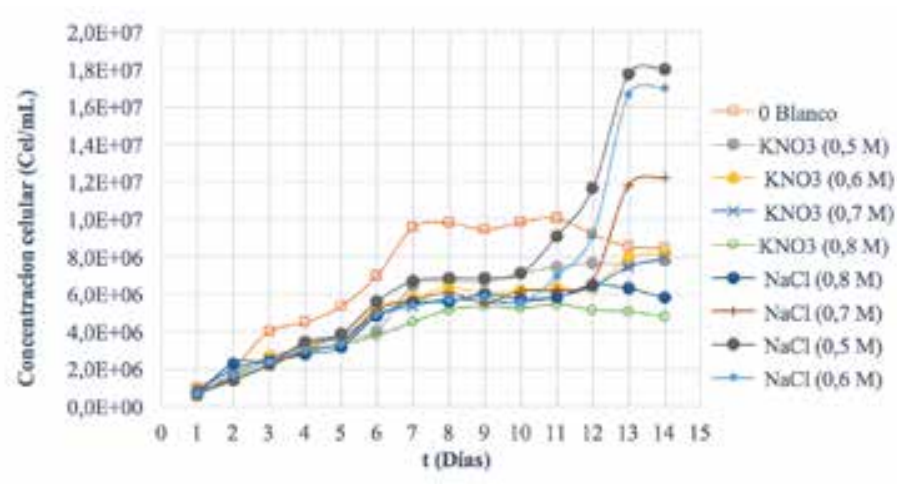

Figura 2. Cambio de la morfología

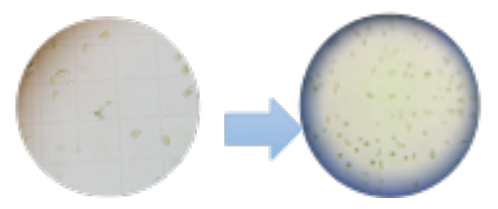

Fuente: elaboración propia.

En la tabla 3 se muestran los resultados de velocidad específica de crecimiento y tiempo de duplicación para cada uno de los cultivos en el pre-experimento. Se observa que la mayor velocidad específica de crecimiento fue alcanzada por las concentraciones de $0,5 \mathrm{M}$ de $\mathrm{KNO}_{3}$ y NaCl , mientras que el mayor tiempo de duplicación corresponde a la concentración $0,8 \mathrm{M}$ para $\mathrm{KNO}_{3}$ y 0,6 $\mathrm{M}$ para $\mathrm{NaCl}$.
Tabla 3. Velocidad específica de crecimiento y tiempo de duplicación preexperimento

\begin{tabular}{ccc}
\hline Sal & $\begin{array}{c}\text { Velocidad específica } \\
\text { de crecimiento } \boldsymbol{\mu}_{\mathrm{e}} \\
\left(\mathbf{d}^{-1}\right)\end{array}$ & $\begin{array}{c}\text { Tiempo de } \\
\text { duplicación } \\
\text { (Día) }\end{array}$ \\
\hline $\mathrm{KNO}_{3}(\mathbf{0 , 5} \mathrm{M})$ & 0,2369 & 2,9253 \\
\hline $\mathrm{KNO}_{3}(\mathbf{0 , 6} \mathrm{M})$ & 0,1912 & 3,6262 \\
\hline $\mathrm{KNO}_{3}(\mathbf{0 , 7} \mathrm{M})$ & 0,1902 & 3,6443 \\
\hline $\mathrm{KNO}_{3}(\mathbf{0 , 8} \mathrm{M})$ & 0,1847 & 3,7538 \\
\hline $\mathrm{NaCl}(\mathbf{0 , 5} \mathrm{M})$ & 0,2728 & 2,5409 \\
\hline $\mathrm{NaCl}(\mathbf{0 , 6} \mathrm{M})$ & 0,2057 & 3,3702 \\
\hline $\mathrm{NaCl}(\mathbf{0 , 7} \mathrm{M})$ & 0,2358 & 2,9396 \\
\hline $\mathrm{NaCl}(\mathbf{0 , 8} \mathrm{M})$ & 0,2096 & 3,3073 \\
\hline $\mathrm{Blanco}$ & 0,2176 & 3,1860 \\
\hline
\end{tabular}

Al realizar el análisis espectrofotométrico a estas muestras, se observó que el cultivo con $\mathrm{KN}_{3} 0,6 \mathrm{M}$, obtuvo la mayor acumulación de carotenoides: 0,3357 $\pm 0,02 \mu \mathrm{g} \mathrm{mL}^{-1}$ como muestra la figura 3 . Al respecto, Príibyl (2016) obtuvo $34 \mu \mathrm{g}$ de carotenoides $\mathrm{mL}^{-1}$ con $\mathrm{KNO}_{3}, 1 \mathrm{M}$. La gran diferencia en estos resultados puede deberse a varios factores: el autor aplicó un estrés combinado nutricional-lumínico, tuvo mejor control de las condiciones del cultivo, o pudo haber modificado la especie genéticamente para convertirla en súper-productora del metabolito de interés. Con relación a los cultivos, con $\mathrm{NaCl}$ la máxima acumulación de carotenoides fue 0,1791 $\pm 0,01 \mu \mathrm{g} \mathrm{mL}^{-1}(\mathrm{NaCl} 0,6$ $\mathrm{M})$, de lo que se puede deducir que la mejor sal para la acumulación de carotenoides en Scenedesmus dimorphus es $\mathrm{KNO}_{3}$, la cual supera la acumulación de carotenoides de los cultivos con $\mathrm{NaCl}$. Como el objetivo del presente trabajo es determinar el mejor factor para acumular carotenoides, se decidió usar la sal $\mathrm{KNO}_{3} 0,6 \mathrm{M}$, a pesar de que no fuera la mejor en cuanto a velocidad específica de crecimiento.

Figura 3. Carotenoides totales de los cultivos con $\mathrm{KNO}_{3}$ y NaCl del pre-experimento

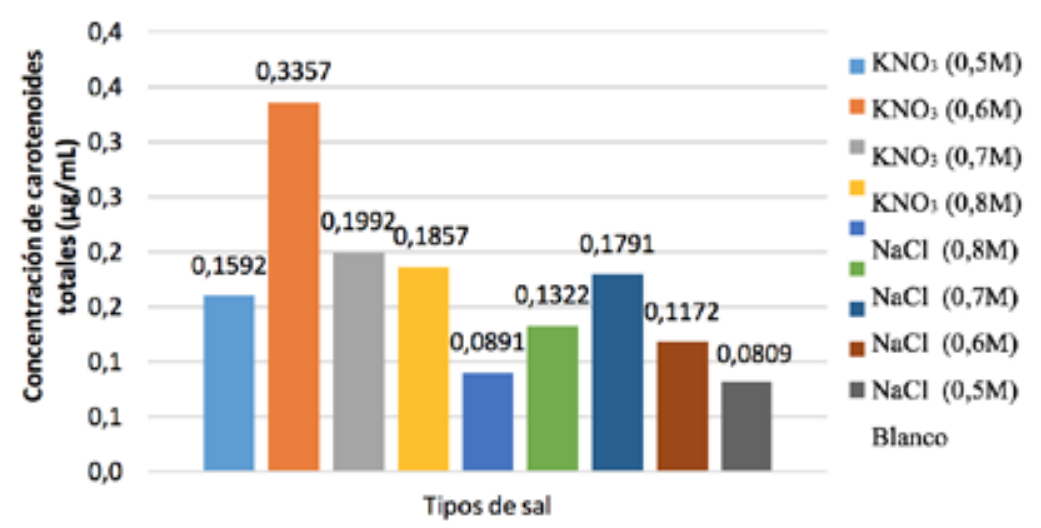


Otro parámetro a tener en cuenta fue el viraje de coloración (figura 4), el cual cambia de verde a amariIlo. Esto se evidencia para la muestra con $\mathrm{KNO}_{3} 0,6 \mathrm{M}$, siendo este cambio un indicativo cualitativo de la presencia de carotenoides.

Figura 4. Viraje de coloración $\mathrm{KNO}_{3}$ a 0,6M pre-experimento

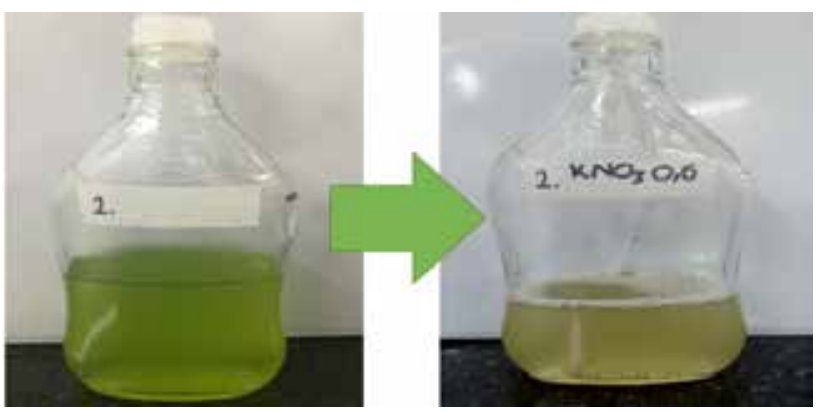

Fuente: elaboración propia.

Al realizar el análisis estadístico se obtuvieron los resultados descritos en la tabla 4, encontrando que no existe una relación entre el factor $\mathrm{A}\left(\mathrm{KNO}_{3}\right)$ y el factor $B(\mathrm{NaCl})$; por ende, no tienen el mismo efecto sobre la acumulación de carotenoides de los cultivos. Igualmente, el factor $\mathrm{B}(\mathrm{NaCl})$ acepta la hipótesis nula, la cual se fundamenta en que la concentración de esta sal no genera un cambio significativo en la concentración de carotenoides totales. Por último, el factor A $\left(\mathrm{KNO}_{3}\right)$ acepta la hipótesis alternativa, puesto que la concentración de la sal genera un cambio significativo en la concentración de carotenoides totales. Estos resultados apoyan la decisión de seleccionar la sal $\mathrm{KNO}_{3}$ como la mejor opción para obtener una mayor acumulación de carotenoides totales en la especie de microalga Scenedesmus dimorphus.

Tabla 4. Análisis por diseño factorial pre-experimento

\begin{tabular}{ccccccc}
\hline Factores & Resultados & $\geq \leq$ & F a $\alpha$ de $\mathbf{0 , 0 5}$ & $v \mathbf{1}$ & $v \mathbf{2}$ & Respuesta \\
\hline FAB & 2,6667 & $>$ & 4,07 & 3 & 8 & $\mathrm{H}_{\mathrm{o}}=$ verdadero \\
\hline FA & 14,0811 & $>$ & 5,32 & 1 & 8 & $\mathrm{H}_{1}=$ verdadero \\
\hline FB & 3,9045 & $>$ & 4,07 & 3 & 8 & $\mathrm{H}_{\mathrm{o}}=$ verdadero \\
\hline
\end{tabular}

Fuente: elaboración propia.

Para la realización del diseño factorial se usó el libro titulado Handbook of tables for probability and statitiscs, específicamente la tabla A.6 (Beyer, 1966).

\section{Resultados del experimento}

Como se mencionó, con base en los resultados del pre-experimento, se escogió la sal $\mathrm{KNO}_{3} 0,6 \mathrm{M}$ para la ejecución del experimento. La figura 5 muestra la curva del crecimiento celular, en la que se ve fase de adaptación del día 1 al 4; es decir, un día adicional con respecto al pre-experimento. La fase exponencial duró del día 5 al 12, menos que en el pre-experimento, debido al aumento en la proporción de nutrientes del medio. Finalmente, del día 12 al 15 se desarrolla la fase estacionaria antes de la aplicación de la sal. Después de adicionar la sal se observó, análogamente al pre-experimento, un aumento ligero de la concentración celular con respecto al blanco. La microalga siguió en fase estacionaria después de dicho aumento.

Figura 5. Curva de crecimiento celular del experimento

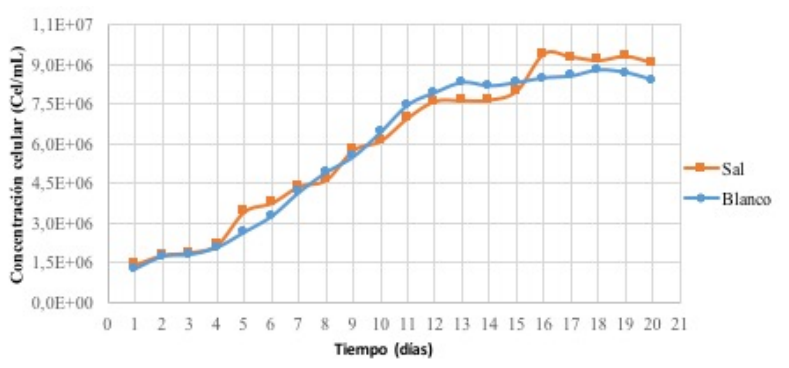

La velocidad de crecimiento para ambos cultivos fue similar: 0,1675 $\mathrm{d}^{-1}$ para el blanco y $0,1564 \mathrm{~d}^{-1}$ para el cultivo con $\mathrm{KNO}_{3} 0,6 \mathrm{M}$. Además, el tiempo de duplicación fue menor para el cultivo blanco $(4,1380$ días $)$ que para el cultivo con sal (4,4322 días).

En cuanto al análisis espectrofotométrico, el cultivo con salinidad alcanzó $0,7395 \pm 0,05 \mu \mathrm{g}$ de carotenoides $\mathrm{mL}^{-1}$, mientras el blanco solamente obtuvo 0,1636 $\pm 0,05 \mu \mathrm{g} \mathrm{mL} \mathrm{m}^{-1}$. Asimismo, se registró un aumento de $0,4038 \mu \mathrm{g}$ de carotenoides $\mathrm{mL}^{-1}$ con respecto a lo obtenido en el pre-experimento.

La máxima productividad de biomasa la presentó el cultivo con $\mathrm{KNO}_{3}, 0,6 \mathrm{M}\left(0,0927 \mathrm{gL}^{-1} \mathrm{~d}^{-1}\right)$, mientras que la del cultivo blanco fue inferior $\left(0,0538 \mathrm{gL}^{-1} \mathrm{~d}^{-1}\right)$. Al comparar ambas productividades con lo reportado por Fonseca y Guarín (2018), quienes emplearon la misma especie de microalga, se puede observar una diferencia significativa, puesto que dichos autores obtuvieron una productividad total de $0,372 \mathrm{~g} \mathrm{~L}^{-1} \mathrm{~d}^{-1}$, aunque empleando reactores de diferentes geometrías. 


\section{Perfil de ácidos grasos}

Por medio de la cromatografía HPLC se encontraron los siguientes porcentajes en masa: $21,1 \%$ de ácidos grasos monoinsaturados (MUFAS); $43 \%$ de poliinsaturados (PUFAS) (34\% omega- 6 de $34 \%$ y omega-3 de $8,9 \%$ ) y $35,9 \%$ de saturados (SFAS). Estos resultados se muestran en la tabla 5 .

Tabla 5. Perfil lipídico del cultivo con $\mathrm{KNO}_{3}$ a $0,6 \mathrm{M}$ en el experimento

\begin{tabular}{cc}
\hline Nombre & \% Ácidos grasos \\
\hline Ácido mirístico (C14:0) & 2,10 \\
\hline Ácido miristoleato (C14:1) & 0 \\
\hline Ácido pentadecanoato (C15:0) & $0 \%$ \\
\hline Ácido pentadecenoato ( C15:1) & 0 \\
\hline Ácido palmítico (C16:0) & 22,50 \\
\hline Ácido palmitoleico (C16:1) & 1,73 \\
\hline Ácido margárico (C17:0) & 1,67 \\
\hline Ácido heptadecenoico (C17:1) & 1,15 \\
\hline Ácido esteárico (C18:0) & 9,60 \\
\hline Ácido elaídico (C18:1n-9t) & 0 \\
\hline Ácido oleico (C18:1n-9c) & 17,80 \\
\hline Ácido cis-vaccenico (C18:1n-7) & 0,40 \\
\hline Ácido linoleico (C18:2n-6c) & 34 \\
\hline Ácido g-linoleico (C18:3n-6) & 0 \\
\hline Ácido linolénico (C18:3n-3) & 8,90 \\
\hline Total & 100 \\
\hline
\end{tabular}

De los ácidos poliinsaturados, el ácido linoléico (omega 6) es el que se encuentra en mayor proporción (34 \%). Por su parte, de los ácidos monoinsaturados, el ácido palmitoleico (omega 7) cuenta con el porcentaje más alto (1,73\%). Por último, del total de ácidos saturados, el de mayor proporción fue el ácido palmítico $(22,5 \%)$.

\section{CONCLUSIONES}

La salinidad fue seleccionada como el factor de estrés propicio para favorecer la generación de carotenoides en la especie de microalga Scenedesmus dimorphus. Luego de evaluar las sales $\mathrm{NaCl}$ y $\mathrm{KNO}_{3}$, se determinó que la que más favoreció la acumulación de carotenoides fue $\mathrm{KNO}_{3} 0,6 \mathrm{M}$. Al realizar el proceso en un volumen de $3,53 \mathrm{~L}$, se obtuvieron $0,7395 \pm 0,05 \mu \mathrm{g}$ de carotenoides $\mathrm{mL}^{-1}$, un notorio aumento con respecto a la concentración del blanco $(0,1636 \pm 0,02 \mu \mathrm{g}$ de carotenoides $\mathrm{mL}^{-1}$ ).
Por otra parte, se produjeron $0,0012 \mathrm{~g}$ en peso seco de carotenoides y 3 gramos en peso seco de lípidos, indicando una proporción de 1:2.500 de carotenoides a lípidos, por lo cual la salinidad generó carotenoides en una proporción muy baja con respecto a los lípidos. A partir de este resultado, se concluye que es poco factible usar este cultivo para la producción a gran escala de carotenoides naturales, aunque no se descarta su aptitud para la producción de lípidos, sobre todo de omega 6, como lo indica el perfil lipídico. Dicho perfil lipídico mostró un porcentaje de 21,1\% de MUFAS, $43 \%$ de PUFAS y $35,9 \%$ de SFAS, con un contenido de omega-6 (34\%) y omega-3 (8,9\%).

Con relación a otros autores, la productividad de biomasa en el presente trabajo fue relativamente baja, siendo $0,0927 \mathrm{gL}^{-1}$ día-1 para $\mathrm{KNO}_{3} 0,6 \mathrm{M}$. Sin embargo, esta aumentó con respecto al blanco, el cual llegó a 0,0538 gL $^{-1}$ día $^{-1}$.

Para futuras investigaciones se recomienda analizar y evaluar la influencia combinada de estrés nutricional y lumínico en la generación de carotenoides totales en la especie Scenedesmus dimorphus.

\section{REFERENCIAS}

Acevedo, A., \& Ramírez, J. (2003). Influencia de un gradiente cruzado de luz y temperatura en la morfología de Scenedesmus acutus meyen var. globosus hortobágyi y sus implicaciones taxonómicas. Actual Biology, 25(79), 141-146.

Beyer, W. H. (1966). Handbook of tables for probability and statistics. Cleveland: The chemical Rubber Co.

Dhaka, P., \& Singh, G. P. (2018). Effect of pH on growth and biopigment accumulation of green alga Dunaliella salina. International Journal of Pharmaceutical Sciences and Research, 9(1), 271276.

Forján-Lozano, E., Garbayo-Nores, I., Casal-Bejarano, C., \& Vílchez-Lobato, C. (2007). Enhancement of carotenoid production in Nannochloropsis by phosphate and sulphur limitation. Communicating Current Research and Educational Topics and Trends in Applied Microbiology. Universidad de Huelva, España. 
Fernández-Cordero, B. (2013). Producción de carotenoides por microalgas y caracterización de la ruta carotenogénica en Chlorella zofingiensis (Tesis doctoral, Departamento de Ingeniería Química). Universidad de Sevilla, Sevilla, España.

Fonseca, L. A., \& Guarín, M. P. (2018). Evaluación de la remoción de cromo con cultivos de microalgas en un fotobiorreactor de panel plano (Tesis de grado, Ingeniería Química). Fundación Universidad de América, Bogotá, Colombia.

Fu, W., Guđmundsson, Ó., Paglia, G., Herjólfsson, G., Andrésson, Ó. S., Palsson, B. Ø., \& Brynjólfsson, S. (2013). Enhancement of carotenoid biosynthesis in the green microalga Dunaliella salina with lightemitting diodes and adaptive laboratory evolution. Applied microbiology and biotechnology, 97(6), 2395-2403.

López, J. A., Fimbres-Olivarría, D., Medina-Juárez, L. A., Miranda-Baeza, A., Martínez-Córdova, M. L., \& Molina-Quijada, D. M. A. (2013). Producción de biomasa y carotenoides de Dunaliella tertiolecta en medios limitados en nitrógeno. Phyton, 82(1), 23-30.

López, Y. K. (2008). Caracterización genética y de metabolitos secundarios de diferentes ais/amientos de Dunaliella salina bajo condiciones de estrés salino (Trabajo de Grado de Maestría en Ciencias en Biotecnología Genómica). Instituto Politécnico Nacional, Universidad de México, México.

Moronta, R., Mora, R., \& Morales, E. (2006). Respuesta de la microalga Chlorella sorokiniana al $\mathrm{pH}$, salinidad y temperatura en condiciones axénicas y no axénicas. Revista de la Facultad de Agronomía, 23(1), 27-41.

Murcia, M., J., L., \& Parra M, M. A. (2018). Producción de proteínas a partir de la microalga Chlorella vulgaris enriqueciendo el medio de cultivo con fuentes de nitrógeno (Tesis de grado, Ingeniería Química). Fundación Universidad de América, Bogotá, Colombia.

Plaza, M., Herrero, M., Cifuentes, A., \& Ibanez, E. (2009). Innovative natural functional ingredients from microalgae. Journal of agricultural and food chemistry, 57(16), 7159-7170.
Přibyl, P., Pilný, J., Cepák, V., \& Kaštánek, P. (2016). The role of light and nitrogen in growth and carotenoid accumulation in Scenedesmus sp. Algal research, 16, 69-75.

Restrepo, T. I., Díaz, G. J., \& Pardo, S. C. (2012). Peces dulceacuícolas como alimento funcional: perfil de ácidos grasos en tilapia y bocachico criados en policultivo. Revista de biotecnología Unicauca, 10(2), 44-53.

Rubio, F., D., Sandoval, J. A., Ruíz, J. P., \& Tovar, J. C. (2017). Escalamiento de un fotobiorreactor a nivel piloto para la producción de biomasa. Revista de Investigación, 10(1), 61-72.

Vega, B. O. A., \& Voltolina, D. (2007). Concentración, recuento celular y tasa de crecimiento. En Métodos y herramientas analíticas en la evaluación de la biomasa microalgal (pp. 17-25). Ciudad de México: CONACYT.

Velázquez, J. (2012). Problemas de salud ocasionados por los aditivos, preservativos, colorantes $y$ sabores artificiales, hormonas y antibióticos en la alimentación industrial del mundo moderno. Universidad Interamericana de Puerto Rico.

Wichuk, K., Brynjólfsson, S., \& Fu, W. (2014). Biotechnological production of value-added carotenoids from microalgae: Emerging technology and prospects. Bioengineered, 5(3), 204-208. 